\title{
La paleodemografía: ¿un instrumento para simular el comportamiento demográfico del pasado? Análisis comparativo con la demografía histórica en la Ciudad de México del siglo XIX
}

\section{Allan O rtega Muñoz*}

En este estudio se emplearon los métodos de evaluación de la información y de análisis demográfico para examinar el comportamiento de dos series poblacionales de la Ciudad de México del siglo XIX que provienen de los restos esqueléticos que fueron exhumados en el Panteón de Santa Paula y de los registros de la Parroquia de Santa María.

Los resultados muestran diferencias entre todos los análisis, y con ellos se construyeron dos escenarios demográficos, pues la demografía histórica refiere una fecundidad mayor que la determinada por la paleodemografía, lo cual ocasiona que los cálculos de la mortalidad sean por tanto disímiles. Estas diferencias provienen de las desiguales distribuciones de edad a la muerte, por lo que carece de sustento la idea de complementar el estudio demográfico de las poblaciones pretéritas con la combinación de estas disciplinas.

Palabras clave: paleodemografía, demografía histórica, análisis demográfico.

Fecha de recepción: 5 de septiembre de 2003.

Fecha de aceptación: 8 de diciembre de 2003.

Paleodemography: an Instrument for Simulating the Demographic Behavior of the Past? Comparative Analysis with the Historical Demography of $19^{\mathrm{h}}$ Century Mexico City

Several methods of information assessment and demographic analysis were used in this study to examine the behavior of two population series in $19^{\text {h }}$ century Mexico City, drawn from the skeletal remains exhumed in the Panteón de Santa Paula and the records from the Parroquia de Santa Maria.

The results, showing differences between all the analyses, were subsequently used to construct two demographic scenarios, since historical demography yields more results than paleodemography, which also leads to certain differences in the calculations of mortality. These differences are due to the unequal distributions of age at death, which effectively refutes the idea of complementing the demographic study of earlier populations through the combination of these disciplines.

Key words: paleodemography, historical demography, demographic analysis.

* Profesor-investigador del Centro Inah Quintana Roo. Correo electrónico: allanortega@yahoo.com 


\section{Introducción}

Tanto la demografía histórica como la paleodemografía se encargan del estudio del comportamiento demográfico de las poblaciones pretéritas, aunque cada una cuenta con una metodología particular y fuentes de información específicas. La demografía histórica combina las herramientas de la demografía y la epistemología de la Historia, y la paleodemografía se acerca al pasado demográfico mediante el análisis demográfico y el marco teórico de la antropología.

El análisis demográfico de las poblaciones humanas del pasado impone diversos retos tanto por su metodología como por su fundamentación teórica. Las dos materias mencionadas abordan el problema en formas distintas, de ahí que ambos fundamentos teórico-metodológicos suelan llegar a diferentes resultados. Es común, por ejemplo, que en diversas publicaciones aparezcan las estimaciones de la esperanza de vida al nacimiento $\left(\dot{e}_{O}\right)$ de la población para diferentes localidades y regiones del México prehispánico, colonial e independiente, obtenidas a partir de ambas disciplinas (Velasco, 1992; Ceja y Hernández, 1998; Márquez, 1998; Gómez de León, 1998), sin que hasta el momento se haya realizado una evaluación consciente de las posibles discrepancias entre ellas.

Más todavía, en los diversos círculos de investigadores se reconoce la necesidad de complementar las inferencias demográficas de una región y tiempo específicos obtenidas por la paleodemografía, con un estudio demográfico-histórico de la misma región y tiempo (Ceja y Hernández, 1998: 136; Hernández, 1999); pero ¿qué tan compatibles pueden ser sus resultados como para hablar de una complementariedad? Por lo tanto, el objetivo idóneo será realizar un análisis comparativo de las fuentes de información de cada una de las disciplinas utilizando métodos de evaluación de la calidad de la información y aplicando las herramientas propias del análisis demográfico y, en este caso, empleando datos de una muestra esquelética amplia y sus correspondientes registros parroquiales del siglo XIX. La intención del artículo que aquí se presenta consiste en realizar una crítica a la paleodemografía y reflexionar sobre el quehacer de los antropólogos en su tarea de reconstruir algunos perfiles demográficos, con el propósito de impulsar el desarrollo certero de estos tipos de investigaciones minimizando los errores metodológicos al emplear simultáneamente ambas disciplinas de la demografía, la antropología y la historia. 
Como hipótesis de trabajo se plantea que el comportamiento demográfico (dado por la observación de las curvas de mortalidad y fecundidad, las esperanzas de vida, la edad media de la muerte, las tasas de mortalidad y natalidad) nos lleva a dos escenarios demográficos diferentes porque las fuentes de datos de cada disciplina miden un comportamiento demográfico distinto, y por ende la paleodemografía y la demografía histórica no cumplen una función de complementariedad para el cabal conocimiento del comportamiento demográfico de las poblaciones del pasado.

\section{Marco teórico}

El estudio comparativo del registro esquelético —arqueológico-con los registros parroquiales ha sido poco trabajado. Un ejemplo es la investigación realizada por Walker y sus colaboradores (1988), quienes al estudiar el cementerio de la Misión La Purísima en California y sus correspondientes registros de la Misión Franciscana de 1813 a 1849 se propusieron evaluar los procesos tafonómicos diferenciales de los restos humanos y no trataron de aplicar ambas metodologías para comprender los hechos demográficos relativos a esa población.

Otro ejemplo es el estudio de Piontek y Weber (1990), donde tras comparar un registro parroquial del siglo xIx y los restos óseos de un cementerio usado entre 1350-1650 en la misma parroquia de Polonia, los autores encontraron que existía una convergencia en el curso de las curvas de los valores de la esperanza de vida conforme a los datos provenientes de la reconstrucción que emprendieron aplicando ambas disciplinas. Aclararon sin embargo que esta convergencia podría ser accidental.

Se trata de un problema metodológico, pues no hay antecedentes de la comparación de dos metodologías, técnicas y marcos teóricos diferentes, como los empleados en la paleodemografía y la demografía histórica. Por ello, es necesario ubicarnos en un punto medio que nos permita analizar las desigualdades y similitudes a las que están sujetas ambas disciplinas; en este punto medio habrá que repensar el objeto y objetivos de estudio de la demografía para ubicar a las dos disciplinas dentro de ese marco y evaluar hasta qué punto cada una responde a las interrogantes planteadas por la demografía.

La demografía es la ciencia formal que estudia a la población en su conjunto, tanto cuantitativa como cualitativamente, en tiempo y 
espacio, por medio de los fenómenos demográficos —-fecundidad, mortalidad y migración-, relacionando ciertas variables cuantitativas, como la edad y el nivel de escolaridad, con variables cualitativas, como el sexo, el estado civil, la ocupación, o la posición socioeconómica; las cuales tienen la capacidad de alterar la composición por sexo, la estructura por edades y el crecimiento de la población.

Una vez establecido qué entendemos por demografía y cuáles son los métodos por los que alcanza sus objetivos, examinaremos cada una de las disciplinas para determinar cómo se relacionan con la demografía clásica.

\section{La demografía histórica}

Para entender los propósitos y el objeto de estudio de la demografía histórica es necesario comprender su relación con los de la demografía, que antes definimos. Para ello cabría la pregunta: ¿Es la demografía histórica una disciplina de la demografía, como se ha asegurado hasta el momento? Louis Henry (1968), uno de los principales exponentes de la demografía histórica, considera que ésta no constituye simplemente una parte marginal de la demografía, sino que es la demografía en sí misma, ya que ésta ha llegado a ser un estudio de las poblaciones humanas en el tiempo. De hecho, gracias a la demografía histórica se han desarrollado y usado nuevos métodos y ha surgido el deseo de entender las condiciones de vida de aquellos hombres y mujeres que vivieron en el pasado y cuya existencia quedó registrada.

La demografía histórica pretende conocer y analizar cuantitativa y cualitativamente los fenómenos demográficos propios de las poblaciones humanas históricas valiéndose de documentos escritos que no necesariamente fueron creados con propósitos demográficos. Su finalidad es medir y descubrir uniformidades en los procesos básicos que engloban estos fenómenos (nacimientos, muertes, movimiento y crecimiento poblacional) para conocer los cambios en tiempo y espacio del tamaño, composición y distribución de la población, explicando las interrelaciones de aquellos factores (biológicos, culturales, sociales, económicos, políticos, psicológicos, entre otros) que ejercen influencia sobre los fenómenos demográficos.

De hecho, metodológicamente hablando un elemento que distingue a la demografía histórica es la técnica de reconstrucción de familias desarrollada por L. Henry y M. Fleury (1956) en su libro Des 
registres paroissiaux à l'histoire de la population. Manuel de dépouillement et d'exploitation de l'état civil ancien, que constituye una guía práctica para el investigador y no propiamente un tratado de demografía histórica. El método consiste esencialmente en vaciar el contenido de todas las actas que figuran en los registros de una parroquia en fichas especiales. Posteriormente se transcribe esta información en fichas de familia, que son un tanto análogas a las que forman parte de los libros modernos del registro civil, para finalmente llevar a cabo los cálculos demográficos elementales (Dupâquier, 1984).

Para entender la demografía histórica y los resultados a los que se llega a partir de sus fuentes de información es necesario aproximarnos a la problemática implícita en las características de su metodología y sus fuentes de información; a ello dedicaremos la siguiente sección.

\section{La problemática en la demografía histórica}

Varias instituciones y muchos investigadores se han interesado en la problemática implícita en el desarrollo de la demografía histórica; ejemplo de ello es la labor del Centro Latinoamericano de Demografía, que al realizar un análisis de la disciplina la consideraba "circunstancial y periférica”, pues el tema nunca se había tratado con propuestas de investigación (Celade, 1975).

De tal forma, las dificultades a las que se enfrentan los investigadores inmersos en el área de la demografía histórica están relacionadas en su mayoría con las fuentes documentales, pues éstas presentan las siguientes limitaciones: $a$ ) dispersión de las fuentes, especialmente las de procedencia religiosa; $b$ ) desaparición de documentos por efectos del tiempo, el descuido o la destrucción premeditada; $c$ ) poco o nulo trabajo archivístico, que ocasiona que los investigadores se pierdan en un universo de información poco accesible; $d$ ) privatización de los documentos por los coleccionistas, lo cual impide la investigación abierta; e) subregistro de algunos segmentos de la población; f) errores en la consignación de la edad de los individuos cuando los encuestados no la sabían; $g$ ) criterios dispares de recolección de los datos de las autoridades civiles y las religiosas; $h$ ) cambio en el significado de los conceptos; por ejemplo, la institución denominada "familia" no tiene la misma concepción en el pasado que en el presente, lo cual acarrea problemas metodológicos (Arretx et al., 1983; Celade, 1975; y Tuirán, 1992). 
Estos señalamientos ponen en alerta al investigador que realiza inferencias de fuentes históricas, pues como advirtió Hollingsworth (1968), los errores y sesgos pueden contaminar los datos demógrafohistóricos, y la mayor dificultad del problema son las fallas en el registro de los eventos a estudiar, como la muerte, el nacimiento o el casamiento de los sujetos.

¿Qué esperamos, por tanto, de la demografía histórica? La respuesta no es sencilla, todo está en la percepción de la historia que tenga el investigador. ¿Historia para qué? ¿Para reivindicar a las poblaciones pasadas viéndolas en un jardín del edén donde "el tiempo de los [...] antepasados [...] aparece como un tiempo de inocencia y de felicidad"? (Le Goff, 1997: 15), o en verdad piensa en una ciencia por medio de la cual puede buscar las respuestas a las interrogantes del presente.

De hecho, algunos especialistas se refieren a la demografía histórica como la triunfadora en la reconstrucción de una fotografía de la vida y la muerte de las poblaciones del pasado; sobre todo es el caso de los estudios europeos sobre la transición demográfica de los siglos XVIII y XIX (Bocquet-Appel y Masset, 1982). Ejemplo de ello es la edición especial de Daedalus de 1968, donde los investigadores confieren a la demografía histórica su debida importancia cuando abordan la transición demográfica y la problemática derivada de la explosión demográfica de los países del tercer mundo. Roger Revelle (1968: 354) resumió esta postura cuando expuso:

Parece urgente el examinar las condiciones históricas y los procesos que existieron antes de la transición demográfica y durante los tempranos estadios del descenso de la fecundidad con la esperanza de aprender algo que pueda ser aplicable al enorme problema de la reducción de las tasas de nacimiento en los países pobres.

En cuanto a la calidad de la información de las fuentes documentales del siglo xix, Maldonado (1976) asegura que en las parroquias de la capital del país falta información respecto a los matrimonios y las defunciones ocurridas a partir de 1860, lo cual obedece a que aunque en 1859 se dictó la Ley Nacional del Registro Civil en la Ciudad de México, el decreto empezó a tener vigencia en 1867, pues durante la primera época las anotaciones de las autoridades civiles eran poco consistentes, y por tanto la única forma de conocer las características demográficas de esa población es consultando la información de los archivos parroquiales. 
Considero que el empleo de una muestra de la población de la Ciudad de México del siglo xix (teniendo en cuenta todos los pormenores que puedan menguar su confiabilidad $)^{1}$ consignada en la información parroquial puede ser útil para elaborar un análisis crítico de la demografía histórica, y que éste habrá de proporcionar resultados importantes para el avance en el conocimiento del comportamiento demográfico pretérito de México.

\section{La paleodemografía}

El paleodemógrafo ideal debe ser un conocedor de los conceptos y los métodos arqueológicos y antropológicos, pero sobre todo de la antropología física. Por ello la paleodemografía ha atraído la atención de los antropólogos interesados en cuestiones culturales relacionadas con la salud, la historia de la vida y la evolución de la biodemografía humana (Meindl y Russell, 1998; Márquez y Hernández, 2001), cuyos objetivos de investigación se centran en la caracterización de los restos de las poblaciones del pasado para dar luz sobre la historia individual y colectiva de esos grupos humanos (Buikstra y Konigsberg, 1985).

Esta disciplina ha emprendido la simulación del comportamiento demográfico valiéndose de la evaluación de uno de sus fenómenos: la mortalidad de los individuos de una población pretérita, sea prehistórica o protohistórica, y ha abarcado poblaciones históricas con el análisis de sus restos mortuorios, ubicados en un tiempo y espacio específicos.

El insumo necesario para desarrollar esta simulación demográfica es la distribución por edad a la muerte de cada uno de los individuos. Ahora bien, para derivar datos demográficos de estos insumos se deben retomar, además de una serie de indicadores básicos (Márquez y Hernández, 2001), los conocimientos de la teoría de las poblaciones estables, los modelos de poblaciones estables, como la estacionaria, y las tablas modelo de vida.

Sin embargo, como era de esperarse, la paleodemografía no ha escapado a la crítica, enfocada sobre todo a su metodología, a la teo-

${ }^{1}$ De las parroquias existentes durante el siglo xix en la Ciudad de México, la que interesa en el presente estudio es la Parroquia de La Asunción, de Santa María la Redonda. Maldonado (1976) observó allí faltantes de 1868 a 1900 en relación con las defunciones, y de 1833 a 1839 y 1863 a 1868 respecto a los matrimonios. 
ría que la sustenta y a las técnicas que emplea para estimar con precisión el sexo y la edad de los individuos. En los siguientes apartados se presenta una revisión de esta crítica.

\section{La problemática y sus respuestas en la paleodemografía}

Cuando se usan poblaciones esqueléticas para realizar inferencias demográficas el problema toral es que no hay información sobre la estructura de edad de los grupos humanos del pasado, como cuando se trabaja con una población viva. Es decir, no se sabe el número de individuos de cada rango de edad que vivieron y murieron en un periodo designado. Sólo se conoce la cantidad de individuos que fallecieron a edades específicas. Sin contar con la composición por edad y sexo de la población en riesgo, es difícil, si no imposible, obtener la mayoría de las medidas demográficas (Horowitz et al., 1988).

Wood y sus colaboradores (1992) han ideado un concepto interesante para abocarse a este problema de la población en riesgo; lo denominan heterogeneidad escondida en el riesgo de morir. Ésta considera que cualquier población esquelética estudiada está constituida por una mezcla desconocida de individuos con diferente susceptibilidad hacia el riesgo de morir, proporcionando una gran heterogeneidad en el interior de la población. Esta heterogeneidad está relacionada con causas genéticas, con diferenciales socioeconómicas, con microvariaciones ambientales y aun con las tendencias temporales de la salud, ya que la mayoría de las series esqueléticas, especialmente las grandes, representan acumulaciones que abarcan periodos de tiempo más o menos prolongados.

Este problema hace virtualmente imposible interpretar en el ámbito agregado las tasas específicas de mortalidad en términos individuales de muerte, lo cual refleja algo inevitable: es imposible obtener de las muestras arqueológicas estimaciones directas de sus tasas demográficas. Tales estimaciones requieren que se conozcan las cifras relativas a la exposición al riesgo de morir, que tampoco pueden conocerse con precisión. Por tanto, debido a su naturaleza, las muertes son una muestra sesgada de todos los individuos de la población que estuvieron vivos a una edad determinada. 


\section{El universo y la muestra}

Para la paleodemografía el cuestionamiento comienza con la selección de la muestra, ya que hay serias dudas de que los individuos estudiados (hombres y mujeres de todas las edades que murieron y fueron enterrados en el sitio) representen a la totalidad de la población por analizar (Konigsberg y Frankenberg, 1992; Wood et al., 1992). Sin embargo se han realizado esfuerzos encaminados a conocer las relaciones de la población con su medio para determinar en qué grado está completa la muestra, cuál fue la temporalidad en que vivió la población, cuáles eran sus asociaciones culturales, para considerar el posible sesgo de ciertos segmentos de la población a ser excluidos, y cuáles eran las relaciones con otros grupos humanos (Civera y Márquez, 1998).

Los factores externos forman parte de estas críticas y tal vez no se han tomado mucho en cuenta, pese a que pueden alterar la distribución de las edades, sesgando por tanto la muestra. Paine (2000) trata de evaluar algunos de estos factores (como los episodios catastróficos: epidemias y desastres naturales), argumentando que una gran proporción de la mortalidad por catástrofes en una población puede semejar la distribución de las personas que estuvieron vivas, ya que incluye a los niños mayores, adolescentes y adultos jóvenes que muestran el perfil típico de la mortalidad. En su trabajo, los efectos de episodios catastróficos son simulados por la proyección del modelo de población y sujeto a ésta, un gran episodio catastrófico (30\% de mortalidad) como parte de una proyección de población a 100 años. Sus resultados muestran efectos importantes en la distribución por edad a la muerte conforme al perfil juvenil y de adultos jóvenes característico de muchas muestras arqueológicas.

\section{La estimación de la edad a la muerte}

Los críticos de la paleodemografía exponen que si bien es cierto que la estimación de edad a la muerte en individuos subadultos (del nacimiento a los 15 años de edad) es bastante exacta, la de los adultos es más bien imprecisa (Boquet-Appel y Masset, 1982), por lo que en forma resumida exponen tres enjuiciamientos acerca de la estimación de la edad en poblaciones pasadas: 1) se tiende a replicar la estructura por edades de la serie de referencia; 2) hay un subregistro de personas mayores de 50 años debido a la metodología empleada, y 3) una 
vez estimada la edad, el método tradicional de asignar individuos a grupos de edades produce estimaciones desviadas de la estructura real por edades (Boquet-Appel y Masset, 1982 y 1985; Konigsberg y Frankenberg, 1992).

Como respuesta a estas críticas la antropología física ha desarrollado una serie de técnicas y metodologías multifactoriales que permiten determinar las edades con mayor precisión y por ende subsanar la subestimación de los segmentos de población no tomados en cuenta. Ciertas metodologías se emplean para estimar las estructuras por edades, como el iteratedage length key y el maximum likelihood, basados en ecuaciones que evitan reproducir la estructura por edades de la población de referencia (Konigsberg y Frankenberg, 1992); asimismo se han desarrollado métodos semiparamétricos para estimar los perfiles paleodemográficos (Müller, Love y Hoppa, 2002).

\section{La teoría de población estacionaria, la edad y la muestra}

El modelo de la población estacionaria (modelo de la teoría de las poblaciones estables) suele aplicarse a la paleodemografía; parte del supuesto de que cierta población está cerrada a la migración (sólo recibe efectivos por nacimientos y sólo sufre pérdidas por muertes), sus tasas de natalidad y mortalidad son constantes y su tasa de crecimiento es de cero. Este supuesto es difícil de sustentar debido a que ninguna población humana se encuentra aislada de las otras (Wood et al., 1992).

Sin embargo, para la paleodemografía la premisa más importante del modelo de población estacionaria es que la edad media a la muerte $\left(\bar{a}_{d}\right)$ es exactamente igual a la esperanza de vida al nacimiento $\left(\dot{e}_{O}\right)$, y en esta equivalencia se basa la mayoría de las estimaciones empíricas de la mortalidad, con lo cual se simplifican los razonamientos. El asumir este tipo de modelo (estacionario) implica que la distribución de las muertes por edad llegará a ser equivalente a la columna de las muertes por edad $\left(d_{x}\right)$, la cual puede ser usada como base para la reconstrucción de una tabla de vida (Johansson y Horowitz, 1986: 235). Cuando una población no es estacionaria, se advierte en la distribución de edades a la muerte, pues ésta es extremadamente sensible a los cambios en la fecundidad, pero no en la mortalidad (Wood et al., 1992; Aguirre, 1998), por lo que la esperanza de vida y la edad media a la muerte son más representativas de la fecundidad que de la mortalidad (Wood et al., 1992). 
Para observar esta relación cabe mencionar lo siguiente. Si en una población estable se mantiene constante la esperanza de vida al nacimiento, la edad media a la muerte puede tomar un amplio rango de valores dependiendo del nivel de fecundidad: el más alto nivel de fecundidad en un modelo de población será el más bajo en la edad media a la muerte (véase el cuadro 1). Esta relación se basa en la forma en que la fecundidad afecta la estructura de edad de la población cerrada. Ahora bien, si se mantiene constante la fecundidad y se permite que la esperanza de vida al nacimiento varíe, la edad media a la muerte no se verá significativamente alterada en un amplio rango de valores medios y bajos (véase el cuadro 2). Este efecto es la base de la teoría de las poblaciones cuasiestables, y lleva a revalorar los efectos de la mortalidad cuando se estima la fecundidad de una población cerrada. Por tanto, la edad media a la muerte es el reflejo de la fecundidad y no de la mortalidad (Johansson y Horowitz, 1986: 239). ${ }^{2}$

\section{Objeciones al uso de la tabla de vida}

Gómez de León (1998: 156) opina que en la paleodemografía el uso de la tabla de vida o mortalidad "se ha erigido como el indicador por excelencia, pues recupera las dimensiones demográficas clásicas de edad y sexo, disponibles en los restos, y asimismo facilita la comparación directa" entre diferentes grupos humanos a través del tiempo y del espacio geográfico en el que se asentaron.

De acuerdo con Civera y Márquez (1998) son tres los problemas que se le han objetado a la utilización de tablas de vida: 1) la posibilidad de que una baja representación de restos infantiles en la muestra pueda modificar los cálculos de la tabla de vida y los valores de sobrevivencia, con lo cual se puede obtener su curva; al respecto,

\footnotetext{
${ }^{2}$ Horowitz et al. (1988) exponen claramente esta relación: si en la dinámica de la población los nacimientos se fueran incrementando constantemente cada año, habría por tanto una mayor proporción de individuos en las categorías de edad más joven que en las de mayor edad. Se calcula que el número de muertes en cada grupo de edad es una proporción de la suma de todos los individuos recobrados, por lo cual en las poblaciones con grandes proporciones en los grupos de edad más jóvenes los cálculos se inclinarán hacia edades tempranas y el resultado será un valor bajo para la edad media a la muerte. Lo contrario ocurrirá cuando los nacimientos decrezcan. Por tanto, es obvio que la edad media a la muerte dependerá de la estructura de la edad, y la tasa de natalidad tendrá un mayor impacto en la estructura de la edad que sobre los cambios equivalentes en la tasa de mortalidad. Por tanto, estos dos factores (tasa de natalidad y estructura por edad) variarán juntos.
} 
CUADRO 1

Edad media a la muerte y fecundidad en cuatro poblaciones con la misma esperanza de vida al nacimiento

\begin{tabular}{cccc}
\hline Población & $\begin{array}{c}\text { Esperanza de vida } \\
\text { al nacimiento }\end{array}$ & $\begin{array}{c}\text { Edad media } \\
\text { a la muerte }\end{array}$ & $\begin{array}{c}\text { Tasa bruta } \\
\text { de reproducción }\end{array}$ \\
\hline P1 & 25 & 25.0 & 2.5 \\
P2 & 25 & 21.5 & 2.9 \\
P3 & 25 & 18.4 & 3.4 \\
P4 & 25 & 15.6 & 3.9 \\
\hline
\end{tabular}

Fuente: Coale y Demeny, 1983, citado por Johansson y Horowitz (1986: 238).

Moore et al. (1975) aseguran que la esperanza de vida y la probabilidad de muerte no se alteran matemáticamente, por lo que se pueden utilizar para realizar interpretaciones válidas; 2) el crecimiento de la población puede constituir un error en las tablas, dado que se asume que se trabaja con poblaciones estables; sin embargo, se ha comprobado que cuando se trata de tendencias a largo plazo estas desviaciones son mínimas (Johansson y Horowitz, 1986); además se han elaborado varias tablas de mortalidad con diversas tasas de crecimiento $(r)$ para seleccionar la que mejor representa a la población, aunque este método ha sido criticado porque no permite la comparabilidad estadística entre poblaciones.

GUADRO 2

U na población con fecundidad constante (tasa bruta de reproducción) y una edad media a la muerte constante, a pesar del aumento de la esperanza de vida al nacimiento y de la tasa de crecimiento

\begin{tabular}{ccccc}
\hline & & & $\begin{array}{c}\text { Esperanza } \\
\text { Te vida }\end{array}$ & $\begin{array}{c}\text { Tasa de } \\
\text { Tiempocimiento/1 000 } \\
\text { por año }\end{array}$ \\
\hline T1 & 3.5 & 17.9 & 20 & 3.6 \\
T2 & 3.5 & 17.6 & 25 & 11.5 \\
T3 & 3.5 & 17.5 & 30 & 17.5 \\
T4 & 3.5 & 17.7 & 35 & 22.5 \\
T5 & 3.5 & 18.5 & 42.5 & 28.5 \\
T6 & 3.5 & 30.8 & 67.5 & 47.5 \\
\hline
\end{tabular}

Fuente: Coale y Demeny (1983), citado por Johansson y Horowitz (1986: 238). 
Calidad de la información de las colecciones esqueléticas del siglo XIX

La calidad de los datos que proporcione una colección esquelética estará en razón de su estado de conservación y del número de las muestras que de ella se obtengan. Las colecciones mexicanas del siglo xix han sido alteradas por los llamados procesos tafonómicos, así como por la continua urbanización de todas las ciudades del país, que ha ido destruyendo el registro y el contexto de las antiguas ciudades del México independiente.

Empero, la colección denominada Santa Paula u Hospital de San Andrés tiene la virtud de haber sido convenientemente excavada en su sitio arqueológico, y su estado de conservación era bueno, lo cual puede asegurarse debido a que más de uno de los esqueletos fue encontrado en los restos de su ataúd, lo que ayudó a que se protegieran de procesos destructivos.

Por ende, la colección ahora estudiada puede arrojar importantes datos sobre los fenómenos demográficos ocurridos en la Ciudad de México durante el siglo xix.

\section{Material y métodos de estudio}

En este apartado presentaremos algunas especificaciones de los materiales que constituyeron la base para la elaboración del presente estudio. Con el afán de lograr su mejor entendimiento lo dividiremos en cuatro secciones: la primera se dedica a la descripción de los materiales arqueológicos del Panteón de Santa Paula; en la segunda se reúnen los datos paleodemográficos; la tercera versa sobre la recopilación de los datos demográfico-históricos, y en la cuarta se presentan algunas técnicas y métodos paleodemográficos y demográfico-históri$\cos$.

\section{Especificaciones arqueológicas del Panteón de Santa Paula}

El material utilizado en el estudio paleodemográfico proviene del siglo xix y fue recuperado en el extinto Panteón de Santa Paula en la Ciudad de México. Este panteón se hallaba en la avenida que actualmente se llama Eje 1 Norte y Paseo de la Reforma, perteneciente a la colonia Guerrero de la Ciudad de México. El material fue extraído 
por medio de un rescate arqueológico cuando se emprendió la construcción de la Línea B del Metro en el año 1995, y en el año 2000, cuando se inició la edificación de una unidad habitacional en la avenida Mosqueta número 42 de la misma colonia. ${ }^{3}$

En un primer momento fue excavado el material proveniente del Panteón de Santa Paula, al cual se denominó con el mismo nombre. En una segunda temporada de excavación se continuó trabajando en el mismo sitio, sin embargo al material esquelético de esta área del panteón se le denominó Hospital de San Andrés. No obstante, considerando que este material pertenece también al panteón se decidió estudiar todos los restos encontrados en la zona como una misma colección. La temporalidad de los materiales arqueológicos está fechada entre principios del siglo XIx hasta mediados del mismo.

\section{Datos paleodemográficos}

Se analizaron todos los individuos, tanto los primarios como los del osario $^{4}$ del panteón, para conformar la muestra un mayor número de ellos.

De esta forma el estudio osteológico se llevó a cabo en 342 individuos. Aunque el sexo sólo pudo determinarse en los adultos, ${ }^{5}$ sí se calculó la edad de todos los esqueletos con el propósito de reconstruir el comportamiento demográfico ${ }^{6}$ de esta población del México

${ }^{3}$ Las excavaciones de 1995 estuvieron a cargo de los arqueólogos Martín González, Cristóbal Valdez y Tomás Villa, y en la excavación del año 2000 participaron los arqueólogos María de Jesús Sánchez, Pascual Tinoco, Alejandro Zúñiga, John Joseph T. y el antropólogo físico Jorge Cervantes.

${ }^{4}$ En los entierros primarios se encuentran los elementos óseos articulados que pertenecen a individuos bien identificados, y en un osario hay un conjunto de elementos óseos desarticulados pertenecientes a varios individuos; allí no se logra identificar a quién corresponde cada elemento (Lagunas y Hernández, 2000). Para la identificación de individuos en el osario se siguió la siguiente metodología: se tomaron todos los coxales de la colección del osario, se enumeraron y se separaron por lado anatómico (izquierdo y derecho) y por sexo, con lo cual se hizo un conteo del número de cada lado, obteniéndose un número máximo de individuos del número mínimo.

${ }^{5}$ Para la determinación del sexo de los individuos se examinaron las características sexuales secundarias observables macroscópicamente en los coxales de los individuos adultos (Bass, 1987: 200-206), considerando que este segmento esquelético es el más confiable como indicador de sexamiento en adultos (Meindl y Russell, 1998). En los individuos subadultos no se han desarrollado estas características, por lo que es imposible determinar su sexo, de ahí que se les clasifique dentro de un grupo especial.

${ }^{6}$ En los individuos subadultos se observaron la emergencia y la erupción dentaria cuando se contó con algunas de las arcadas dentales de los individuos primarios (Bass, 
independiente. En el cuadro 5 se muestra el número de individuos por sexo y grupos de edad.

\section{Datos para la demografía histórica}

Para el estudio de la demografía histórica se decidió estudiar los registros parroquiales bautismales y de defunciones de La Asunción de Santa María la Redonda correspondientes a los años de 1840-1849 y que fueron depositados en el Archivo General de la Nación. La decisión de tomar los datos de esta década obedece a que no se presentaron entonces factores de crisis demográfica que pudieran alterar los resultados (véase el cuadro 3), como epidemias o hambrunas que modifican la tendencia de los nacimientos y de las defunciones. Solamente la invasión de Estados Unidos en 1847, y las epidemias de cólera morbus, tifo y tifoidea en 1848, pudieron alterar la tendencia de los fenómenos demográficos.

Para los datos bautismales se obtuvieron los registros de 1842 a 1849, pues se perdieron los de los años de 1840 y 1841. En total se recabó la información de 1676 individuos bautizados y por lo tanto nacidos entre los años ya mencionados (véase el cuadro 6). Se considera que nacieron en esos años porque se solía bautizar a los niños entre el nacimiento y los primeros cinco días después de haber nacido. Para las defunciones se obtuvieron los datos de los años 1840 a 1849, y fue recabada la información de 2067 individuos (véase el cuadro 7).

Hasta aquí cabe realizar una apreciación sobre ambas fuentes de datos para realizar nuestra labor de crítica y reflexión sobre la paleodemografía. El tomar las inhumaciones de un panteón y los registros de defunciones de la parroquia, ${ }^{7}$ no implica suponer una estricta correspondencia entre ambas fuentes. Sin embargo se buscó que los registros se hubieran llevado a cabo en una época en que el panteón estuvo en uso y que tuvieran una "similar correspondencia"

1987: 289-290). En los casos recuperados del osario se aplicaron, mediante la medición de la longitud diafisiaria de los huesos largos de las extremidades, ecuaciones de regresión lineal (Ortega, 1998). Para la estimación de la edad de los individuos adultos se empleó la superficie auricular del ilium (Lovejoy et al., 1985). En los casos en que no se presentó este elemento óseo, se empleó la metodología que examina el cierre de las suturas exocraneales (Meindl y Lovejoy, 1985).

${ }^{7}$ Las inhumaciones de las defunciones registradas en la parroquia se realizaron en el Panteón de Santa Paula, que se ubicaba en los límites de la parroquia. 
CUADRO 3

Factores de crisis demográfica durante los años de 1800 a 1876

\begin{tabular}{|c|c|c|}
\hline Año & Guerras & Epidemias y hambrunas de 1800 a 1855 \\
\hline $\begin{array}{l}1800-1802 \\
1803 \\
1810 \\
\text { y siguientes }\end{array}$ & & $\begin{array}{l}\text { Tabardillo. } \\
\text { Sarampión. }\end{array}$ \\
\hline $1812-1813$ & Guerra de Independencia & $\begin{array}{l}\text { "Misteriosas fiebres del año trece", } \\
\text { ocasionadas por el paludismo. } \\
\text { El tifo, la tifoidea y la disentería, } \\
\text { exacerbadas por el hacinamiento, } \\
\text { el hambre y la falta de vivienda. }\end{array}$ \\
\hline $1813-1814$ & & $\begin{array}{l}\text { La viruela, iniciada en Veracruz, llega } \\
\text { a la Ciudad de México. }\end{array}$ \\
\hline 1821 & & \\
\hline 1825 & & Epidemia de sarampión. \\
\hline 1829 & Invasión de España & \\
\hline 1833 & & $\begin{array}{l}\text { Epidemia de cólera morbus iniciada } \\
\text { en Tampico, en agosto llega } \\
\text { a la Ciudad de México. }\end{array}$ \\
\hline 1834 & $\begin{array}{l}\text { Guerra civil entre } \\
\text { federalistas-centralistas, } \\
\text { y liberales y conservadores }\end{array}$ & \\
\hline 1838 & Invasión francesa & Epidemia de tifo. \\
\hline 1847 & Invasión de Estados Unidos & \\
\hline 1848 & & $\begin{array}{l}\text { Nueva epidemia de cólera morbus, } \\
\text { tifo y tifoidea. }\end{array}$ \\
\hline 1850 & & Nueva epidemia de cólera morbus. \\
\hline $1850-1860$ & & $\begin{array}{l}\text { Difteria, escarlatina y paludismo } \\
\text { en la Ciudad de México. }\end{array}$ \\
\hline $1853-1855$ & & Continúan los brotes de cólera. \\
\hline 1857 & & $\begin{array}{l}\text { Se registran casos de cólera } \\
\text { en el mes de septiembre. }\end{array}$ \\
\hline 1858 & & Epidemia de sarampión. \\
\hline 1861 & Guerra de Reforma & Epidemia de tifo exantemático. \\
\hline 1865 & & Epidemia de difteria. \\
\hline 1867 & & Epidemia de tifo. \\
\hline 1874 & & Epidemia de tifo hasta 1875. \\
\hline 1874 & & Epidemia de viruela hasta 1877. \\
\hline 1876 & & Exacerbación del paludismo. \\
\hline
\end{tabular}

Fuentes: Bustamante (1982: 417-423), Florescano y Menegus (2000: 399), Márquez (1992: 86), Rodríguez (1986: 92) y Velasco (1992: 102). 
CUADRO 4

Población mexicana según el censo de la región y los estados que la componen, 1838-1857

\begin{tabular}{|c|c|c|c|c|c|c|}
\hline \multirow{2}{*}{$\begin{array}{l}\text { Región } \\
\text { y estado }\end{array}$} & \multicolumn{3}{|c|}{$\begin{array}{c}1838 \\
\text { Gómez de la Cortina } \\
\end{array}$} & \multicolumn{3}{|c|}{$\begin{array}{c}1857 \\
\text { Hermosa } \\
\end{array}$} \\
\hline & Población & $P P T$ & ТСРP & Población & $P P T$ & $T C P P$ \\
\hline Centro & 1389520 & 19.7 & 0.5 & 1512554 & 18.4 & 0.5 \\
\hline México & & & & 1012554 & 12.2 & \\
\hline $\mathrm{DF}$ & & & & 230000 & 2.8 & \\
\hline Guerrero & & & & 270000 & 3.3 & \\
\hline
\end{tabular}

Notas: PPT: Porcentaje de población total de la población nacional total en la unidad geográfica específica, sea región o estado para ese año. TCPP: Tasa de crecimiento porcentual promedio: revela la tasa a la que está creciendo cada región y estado sobre una base anual.

Fuente: Kicza (1993).

\section{CUADRO 5}

Esqueletos por grupos de edad y sexo para la paleodemografía. Panteón de Santa Paula, siglo xix

\begin{tabular}{ccccc}
\hline Grupos de edad & Hombres & Mujeres & Total & $\begin{array}{c}\text { Subadulto sexo } \\
\text { indeterminado }\end{array}$ \\
\hline$<1$ & & & & 38 \\
$1-4$ & & & & 71 \\
$5-9$ & & & 20 & 9 \\
$10-14$ & 1 & 1 & 12 & 1 \\
$15-19$ & 4 & 7 & 27 & \\
$20-24$ & 15 & 12 & 28 & \\
$25-29$ & 16 & 12 & 38 & \\
$30-34$ & 21 & 17 & 49 & \\
$35-39$ & 21 & 28 & 30 & \\
$40-44$ & 15 & 15 & 11 & \\
$45-49$ & 5 & 5 & 1 & \\
$50-54$ & 1 & 0 & 5 & \\
$55-59$ & 3 & 2 & 203 & \\
$60-64$ & 104 & 99 & & \\
\hline Total & Total de individuos estudiados: 342 & \\
& & &
\end{tabular}

Fuente: Cálculos propios. 
CUADRO 6

Año de bautizo y sexo para la demografía histórica. Parroquia de Santa María la Redonda, siglo xIX

\begin{tabular}{cccc}
\hline Año de nacimiento & Hombres & Mujeres & Total \\
\hline 1842 & 38 & 37 & 75 \\
1843 & 89 & 134 & 223 \\
1844 & 108 & 101 & 209 \\
1845 & 105 & 123 & 228 \\
1846 & 114 & 143 & 257 \\
1847 & 103 & 115 & 218 \\
1848 & 97 & 98 & 195 \\
1849 & 129 & 142 & 271 \\
$1842-1849$ & 783 & 893 & 1676 \\
\hline
\end{tabular}

Total de individuos estudiados: 1676

Fuente: Cálculos propios.

CUADRO 7

Defunciones por grupos de edad y sexo para la demografía histórica. Parroquia de Santa María la Redonda, siglo xıx (1840-1849)

\begin{tabular}{crrr}
\hline Grupos de edad & Hombres & Mujeres & Total \\
\hline$<1$ & 349 & 275 & 624 \\
$1-4$ & 476 & 460 & 936 \\
$5-9$ & 77 & 94 & 171 \\
$10-14$ & 21 & 19 & 40 \\
$15-19$ & 20 & 26 & 46 \\
$20-24$ & 28 & 49 & 77 \\
$25-29$ & 24 & 50 & 74 \\
$30-34$ & 46 & 54 & 100 \\
$35-39$ & 32 & 29 & 62 \\
$40-44$ & 44 & 67 & 111 \\
$45-49$ & 20 & 22 & 42 \\
$50-54$ & 27 & 58 & 85 \\
$55-59$ & 9 & 12 & 21 \\
$60-64$ & 35 & 61 & 97 \\
$65-69$ & 7 & 9 & 16 \\
$70-74$ & 21 & 34 & 55 \\
$75-79$ & 8 & 4 & 12 \\
$80-84$ & 7 & 12 & 19 \\
$85+$ & 7 & 10 & 17 \\
Total & 261 & 1346 & 2607 \\
& & &
\end{tabular}

Total de individuos estudiados: 2067

FuENTE: Cálculos propios. 
en cronología con el tiempo de inhumación de los entierros excavados, dado el informe y el contexto arqueológico de los mismos. Empero, y una vez más, no se espera una estricta y total correspondencia, pues no se tiene la seguridad de que esos individuos enterrados pertenezcan a los registros de defunciones consultados en el Archivo General de la Nación y que pertenecieron a la Parroquia de Santa María la Redonda.

Técnicas y métodos paleodemográficos y demográfico1-históricos

El análisis comparativo de las disciplinas se divide en dos apartados: el de la evaluación de los datos y el del análisis demográfico. Para evaluar la posible atracción en alguno de los dígitos se decidió emplear el índice de Myers, y para estimar la representatividad de los sexos de la muestra se empleó el índice de las Naciones Unidas (Wunsch, 1992). Otro método exploratorio es el logito de Brass, empleado por Gómez de León (1998), cuyos resultados fueron satisfactorios.

La evaluación demográfica de los datos se obtuvo mediante la aplicación de la tasa de mortalidad infantil, la tasa bruta de mortalidad, la edad media a la muerte, la esperanza de vida a partir de la creación de la tabla abreviada de mortalidad, la tasa bruta de natalidad y las curvas de mortalidad y fecundidad a partir de los datos obtenidos (Márquez y Hernández, 2001; Ortega, 2002).

Para la elaboración de los datos de mortalidad se empleó una tasa de crecimiento porcentual promedio anual de $0.5 \%$ reportada por Kicza (1993) para la región centro del país durante el periodo 1838-1857 (véase el cuadro 4), ${ }^{8}$ pues se considera que esa región, pese a que logró cierta preeminencia por su importancia en la economía y el comercio, no tuvo un incremento importante en su población pues mantuvo casi la misma cantidad desde 1810 hasta 1862 y se incrementó irregular pero constantemente en la última mitad del siglo. Kicza (1993) asegura que durante el siglo XIX no ocurrió una migración sustantiva desde o hacia México, por lo que las cifras de las tasas de crecimiento porcentual promedio $(\mathrm{TCPP})^{9}$ de la población nacional

${ }^{8}$ Se decidió hacer las tablas abreviadas de mortalidad para ambos sexos debido a la imposibilidad de la paleodemografía de determinar el sexo en los individuos subadultos, y de esta forma hacer comparables las tablas para ambas disciplinas.

${ }^{9}$ El cambio absoluto se calcula al restar de la población de un año de la del siguien- 
se aproximan mucho a la tasa natural de crecimiento de $0.9 \%$ entre 1810 y 1895 . Aunque la Ciudad de México fue la metrópoli más grande del país en el siglo xix, nunca contuvo más de $4.1 \%$ de la población nacional.

\section{Resultados y discusión}

\section{Evaluación de la información}

Índice de Myers

Con el índice de Myers se obtuvo la atracción en los dígitos, por lo que en la paleodemografía (Panteón de Santa Paula) se observa una preferencia en los dígitos $0(0.11), 4(0.007), 5(0.07)$ y $6(0.07)$ y un mayor rechazo en los dígitos $1(-0.093)$ y $9(-0.10)$. En cuanto al análisis en la demografía histórica (Parroquia de Santa María la Redonda) la preferencia de dígitos existe en $0(0.38)$ y 5 (0.018). El rechazo se presenta en mayor nivel en los dígitos $1(-0.73), 3(-0.064), 7(-0.066)$ y $9(-0.060)$.

McCaa (2000) reporta que la preferencia del dígito en la estimación de la edad por parte de los osteólogos está en los dígitos 0 y 5 . Sin embargo la preferencia hacia cierto dígito en las poblaciones esqueléticas se debe tomar como un sesgo intra-observador. En la demografía histórica se empleó este método para analizar la preferencia de dígito en un padrón del siglo xviII, y se encontró atracción en los dígitos $0,8,6$, y 5 y un mayor rechazo en los dígitos 1,3 y 7 (Chena, 1992: 174). La preferencia de dígito en el informe de la demografía histórica variará dependiendo del momento sociohistórico de que se trate, pues refleja la idiosincrasia de la gente que vivió y murió en el siglo xix. Las diferencias que existen en la preferencia y rechazo

\footnotetext{
te en que se disponga de una cifra confiable. Esta diferencia se divide entre la población total del primer año y después entre el número de años que separan a ambas fechas. El número resultante, cuando se expresa en términos porcentuales, es el TcPP del intervalo que media entre los dos años. Como no hay manera de saber qué fluctuaciones pudieron ocurrir en los años intermedios, este acercamiento parece ser el mejor. El cálculo lineal, expresado como тСРp, para llegar a nuevas cifras de la tasa de crecimiento, puede ser una de las mejores técnicas para conocer cómo crecía la población en el tiempo analizado, ya que, como advierte Kicza, siendo aún poco conocidas las historias estatales y regionales del México decimonónico, este promedio tiene menor probabilidad de transmitir inexactitudes imprevistas (Kicza, 1993: 219, 229).
} 
de dígitos entre ambos estudios son tangibles, por lo que cabría esperar en los cálculos posteriores discrepancias entre ellos.

\section{Índice de Naciones Unidas}

Con el índice de Naciones Unidas se obtiene un índice combinado cuyo valor, si es mayor que 40, se "traduce [como] la existencia de una estructura por edad y sexo muy defectuosa" (Wunsch, 1992: 196). Para el Panteón de Santa Paula no se logró obtener el índice combinado debido a la falta de datos por sexo en los primeros grupos de edad ( 0 a 15 años) y a la ausencia de individuos con más de 55 años. Empero, la relación de hombres y mujeres (índice de masculinidad) se presenta de la siguiente manera: hay mayor número de defunciones de hombres que de mujeres en los grupos 25-29, 35-39 y 60-64. Observamos que esos hombres estaban en la etapa productiva de mayor actividad, por lo que su mayor cantidad en la primera mitad del siglo xix puede estar ligada a actividades bélicas, como las que se desarrollaron durante todo el siglo.

En los grupos de 20 a 24 y 40 a 44 años hay una mayor cantidad de mujeres que de hombres, hecho que puede estar ligado al comienzo de la etapa reproductiva, pues la edad media de las mujeres al matrimonio (primeras nupcias) para la Ciudad de México en el año de 1855 fue de 22.1 años de edad (Pescador, 1992a: 166; véase el cuadro 11). Sus fallecimientos se relacionan con los problemas que suelen presentarse durante y después de la maternidad, que aumentan las probabilidades de muerte para las mujeres, y que son, por tanto, un sensible indicador de las condiciones socioeconómicas en que vi-

\section{CUADRO 8}

\section{Análisis demográfico de la paleodemografía y la demografía histórica}

\begin{tabular}{lcc}
\hline Indicadores & $\begin{array}{c}\text { Panteón } \\
\text { de Santa Paula }\end{array}$ & $\begin{array}{c}\text { Parroquia } \\
\text { de Santa María } \\
\text { la Redonda }\end{array}$ \\
\hline Tasa de mortalidad infantil & $180.95 \% 0$ & $373.05 \% 0$ \\
Tasa bruta de mortalidad & $41.90 \% 0$ & $61.69 \%$ \\
Edad media a la muerte & 23.07 años & 15.34 años \\
Esperanza de vida al nacimiento & 23.71 años & 17.13 años \\
Tasa bruta de natalidad & $46.90 \% 0$ & $66.69 \%$ \%o \\
\hline
\end{tabular}

Fuente: Cálculos propios. 


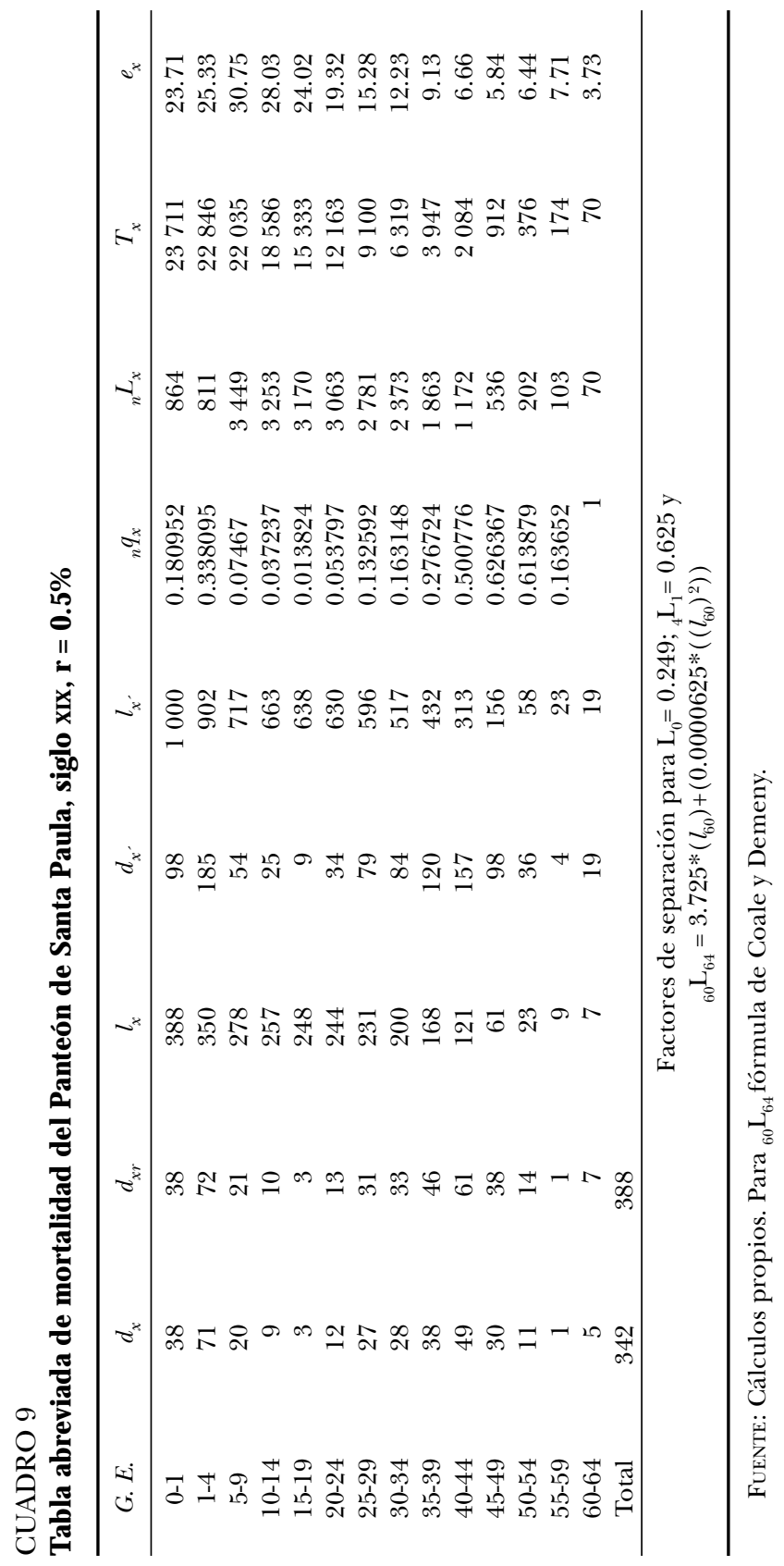




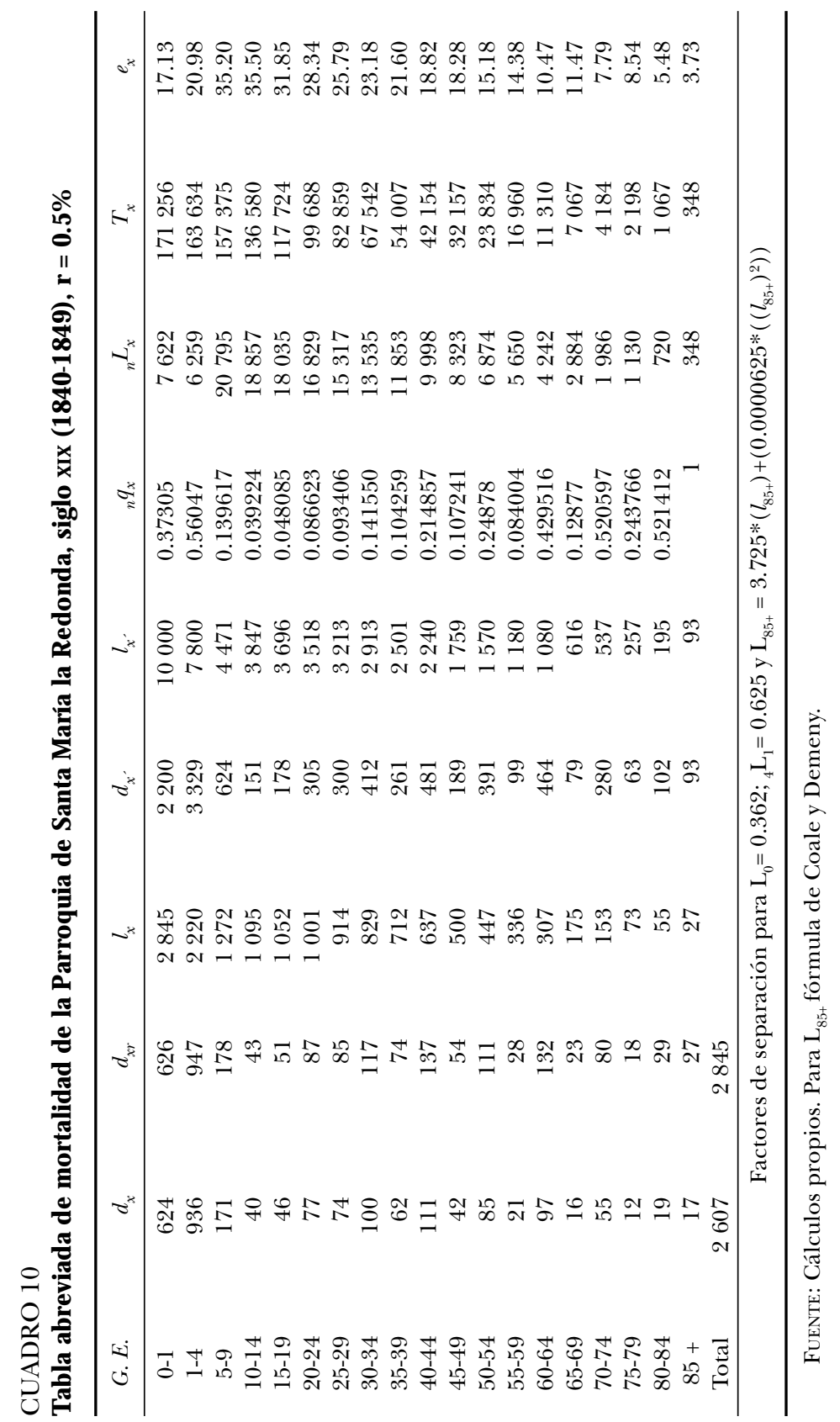




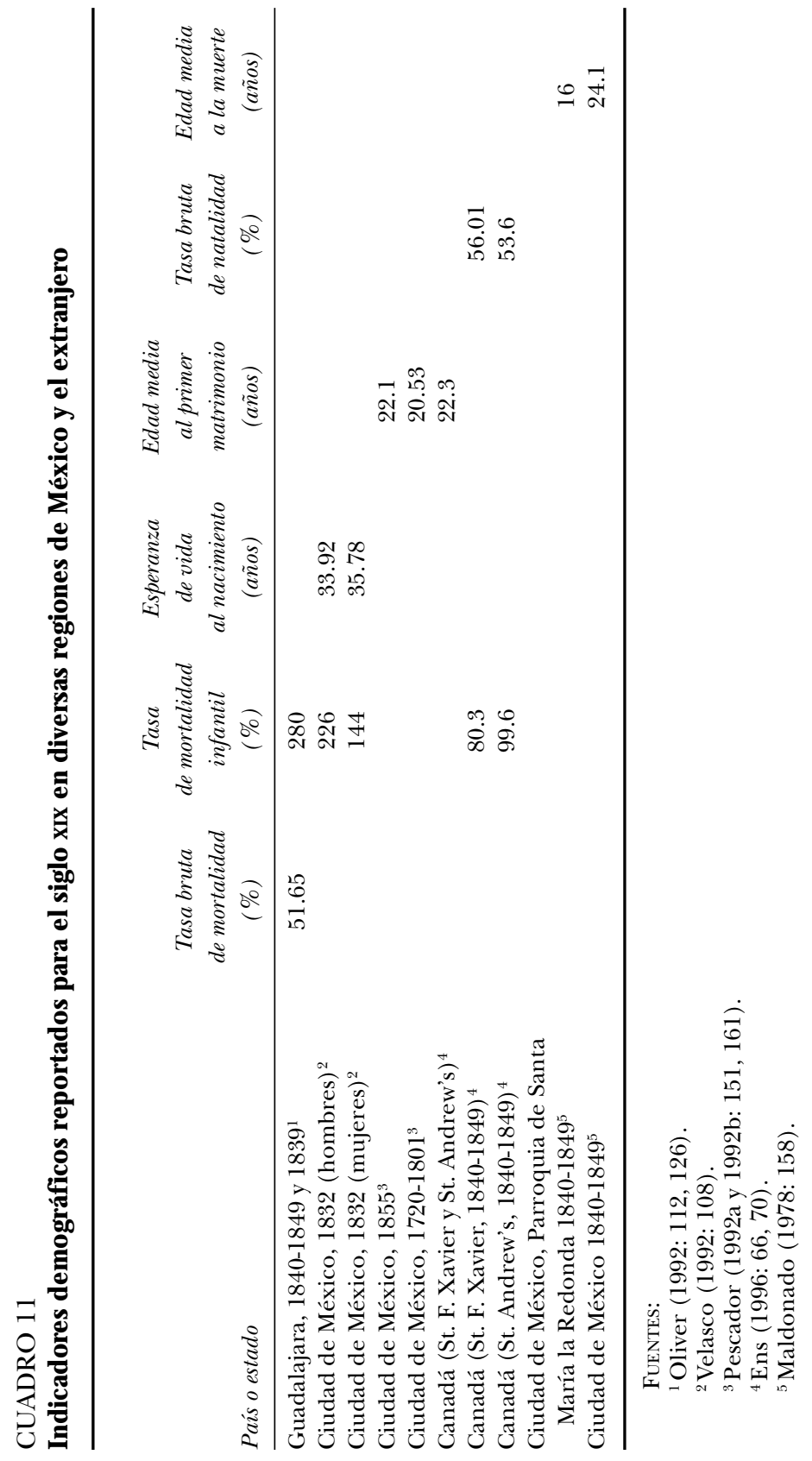


vía la gente. En los grupos de edad restantes se advierte una frecuencia similar de hombres que de mujeres.

Para la Parroquia de Santa María la Redonda el índice combinado es de 270.79, por lo que se podría pensar que en su información sobre defunciones existe una estructura por edad y sexo defectuosa. Sin embargo, hay un mayor número de defunciones de hombres que de mujeres en los grupos de edad: 0-4, 10-14 y 35-39, mientras en los demás hay más defunciones de mujeres que de hombres. La interpretación de esta relación puede ser la misma que en el caso de la paleodemografía.

Sistema logito de Brass

En 1998 Gómez de León propone el método desarrollado por William Brass (1977) como un sistema exploratorio para la identificación de sesgos en la estimación de la edad en las colecciones esqueléticas, ya que éstos pueden alterar el análisis demográfico posterior.

El sencillo análisis consistió en graficar los logitos estándar contra los logitos observados de la colección osteológica del Panteón de Santa Paula como de las defunciones ocurridas en la Parroquia de Santa María la Redonda. Y como estableció Gómez de León (1998: 161): "De acuerdo con el modelo de base, $Y_{(x)}=a+b Y s_{(x)}$, los puntos de tal mapeo deberían ajustarse a una línea recta”.

\section{GRÁFICA 1}

\section{Sistema Logito de Brass para el Panteón de Santa Paula y la Parroquia de Santa María la Redonda, siglo xix, con $r=0.005$}

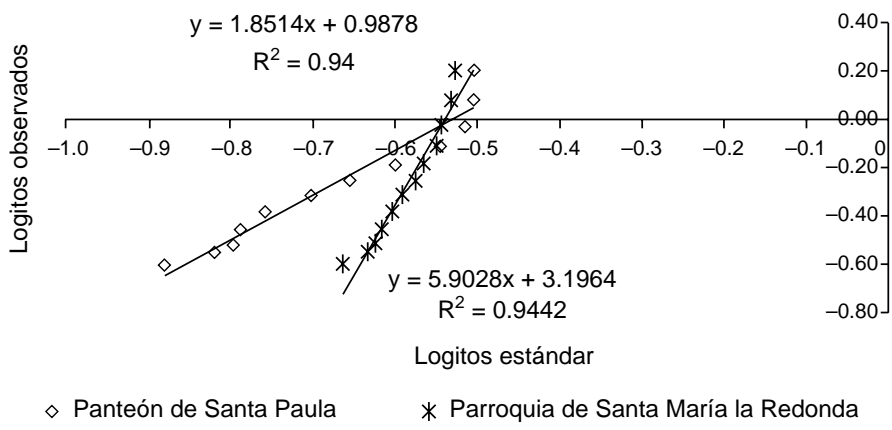


En la gráfica 1 observamos que los datos provenientes del Panteón de Santa Paula se sitúan paralelamente a los logitos estándar de Brass, mientras que los de la Parroquia de Santa María la Redonda el ajuste de los datos a la regresión no se de en los mismos términos que los de la primera disciplina, por tanto presenta algunos problemas en las características de la "selectividad de los datos" (Gómez de León, 1998: 163) y por ende las diferencias se encuentran en la captación de la información de las distribuciones de edades a la muerte, llevándonos a diferentes derroteros.

Análisis demográfico de las dos disciplinas

Mortalidad

Como se expuso en el marco teórico, aunque la mortalidad no se estima partiendo directamente de los datos paleodemográficos, ya que se desconoce la distribución por edad y sexo de la población total, se puede llegar a conocer indirectamente a partir de los indicadores empleados en este trabajo. De esta forma en el cuadro 8 se presentan las tasas de mortalidad infantil, la tasa bruta de mortalidad, la edad media a la muerte y la esperanza de vida al nacimiento, y en los cuadros 9 y 10, las tablas abreviadas de mortalidad para ambos sexos del panteón y de la parroquia.

Notoriamente la tasa de mortalidad infantil y la tasa bruta de mortalidad calculadas para la Parroquia de Santa María la Redonda (demografía histórica) son mayores que las del Panteón de Santa Paula (paleodemografía) (373.05\% y $61.69 \%$ vs. $180.95 \%$ y $41.90 \%$; véase el cuadro 8); empero, la edad media a la muerte es mayor en el análisis paleodemográfico que en el de la demografía histórica (23.07 años vs. 15.34 años; véase el cuadro 8).

Las tasas de mortalidad (infantil y bruta) y la edad media a la muerte, calculadas tanto para el panteón como para la parroquia podrían concordar con las reportadas por diversos investigadores para el siglo xIx, tanto en la Ciudad de México como en otras regiones del país y del extranjero (véase el cuadro 11), por lo que podrían reflejar de manera adecuada el comportamiento demográfico — en la mortalidad- de las poblaciones del pasado. Sin embargo las diferencias entre ambas disciplinas nos llevan a cuestionar si una de las dos estimaciones es la correcta. Tal vez no exista una respuesta, pues ambas 
GRÁFICA 2

\section{Curvas de mortalidad del Panteón de Santa Paula y la Parroquia de Santa María la Redonda, siglo xix, con $r=0.005$}

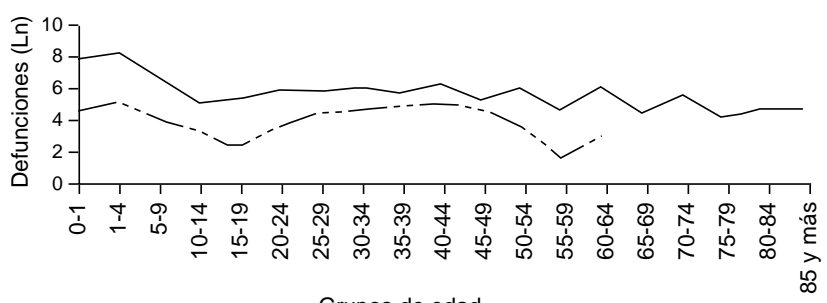

Grupos de edad

__ Panteón de Santa Paula _ _ _ _ Parroquia de Santa María la Redonda

parten de dos fuentes de datos diferentes y cada una tiene sus propios errores y subregistros de la población que alteran los cálculos de mortalidad, por lo que la respuesta sólo podrá hallarse en el contexto sociohistórico de la comunidad estudiada.

En la tabla abreviada de mortalidad, la esperanza de vida al nacimiento calculada para la muestra del Panteón de Santa Paula es mayor que la calculada para la Parroquia de Santa María la Redonda (23.71 años vs. 17.13 años; véase los cuadros 9 y 10). La esperanza de vida calculada para años previos, 1832 en el estudio de Velasco (1992) (véase el cuadro 11), es cercana a la que en la década de 1940 fue calculada para la Ciudad de México a partir de los restos óseos del panteón. Pero, ¿cuál es el origen de la diferencia entre ambos estudios? Éste puede encontrarse en los tamaños de las muestras y en la composición de los grupos de edad de cada una de las fuentes de datos, sobre todo para los primeros grupos de edad, que están subrepresentados en la muestra del panteón y que presentan por tanto una sobrevaloración en su esperanza de vida. Por ello en las esperanzas de vida al nacimiento hay una diferencia de 6.58 años entre ambos estudios, y donde se presenta la mayor desigualdad, de 12.47 años, es en el grupo de 35 a 39 años de edad.

Las curvas de mortalidad expuestas en la gráfica 2 reflejan la distribución de la frecuencia de fallecimientos por grupos de edad. El comportamiento de la mortalidad es similar para ambos estudios, sin embargo el de la paleodemografía está por debajo del de la demografía histórica, hecho que también se advierte en el estudio de Piontek y Weber (1990) donde se grafican las esperanzas de vida al nacimiento 
derivadas de los datos procedentes de un cementerio polaco y de los registros parroquiales que pertenecieron a ese cementerio.

\section{Fecundidad}

Tasa bruta de natalidad y curva de fecundidad. La tasa bruta de natalidad que aparece en el cuadro 8 muestra valores más altos en los cálculos derivados de la demografía histórica que en los que provienen de la paleodemografía (66.69 vs. 46.90\%o y las tasas de natalidad son comparables con las que ofrecen otros estudios (véase el cuadro 11).

En las curvas de fecundidad de la gráfica 3 se aprecian las diferencias en las calculadas para ambos estudios. El calendario de la fecundidad es similar, pero no así la intensidad, pues el cálculo de la demografía histórica alcanza mayores valores que el de la paleodemografía. Sin embargo tanto la intensidad como el calendario de la fecundidad concuerdan con la nupcialidad determinada por Pescador (1992a y 1992b) para la Parroquia de San Miguel de la Ciudad de México en 1855 y para la Ciudad de México en los años 1720 a 1801. Este autor coligió que la edad media al primer matrimonio se presentaba a los 22.1 años para los hombres y 20.53 para las mujeres (cuadro 11). La similitud se debe a que fueron obtenidos empleando la misma metodología a partir de los coeficientes de Weiss (Márquez y Hernández, 2001); sin embargo, las diferencias de intensidad pueden atribuirse al tamaño de las muestras de los grupos de edad en edades reproductivas de cada fuente de información.

GRÁFICA 3

Curvas de fecundidad del Panteón de Santa Paula y la Parroquia de Santa María la Redonda, siglo xIx, con $r=0.005$

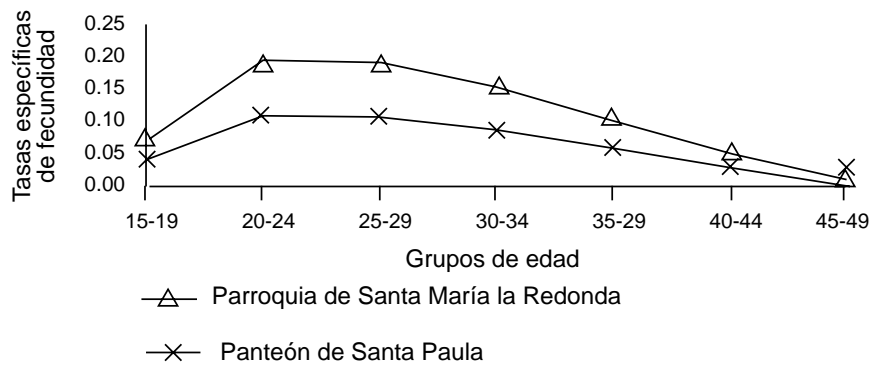




\section{Conclusiones}

La paleodemografía y la demografía histórica son dos disciplinas de la demografía que a lo largo del tiempo han crecido teórica y metodológicamente, sustentadas en los supuestos de las poblaciones estables. Ambas son capaces de recrear el comportamiento demográfico de las poblaciones del pasado a partir de diferentes fuentes de información (documentos históricos y colecciones esqueléticas) con el afán de dar respuesta a las interrogantes de la demografía clásica. Sin embargo, el análisis exploratorio de los datos paleodemográficos y de la demografía histórica nos muestra que existen diferencias entre ellos en la atracción de dígito, debido en primer lugar al sesgo del investigador al momento de estimar la edad de los esqueletos en el caso de la paleodemografía, y en segundo lugar, a la posiblemente "falsa" declaración de la edad que hicieran el familiar del difunto o la persona que registró las edades de los que murieron en el pasado, en este caso los vecinos de la Parroquia de Santa María la Redonda, en la Ciudad de México del siglo xix.

La razón de las diferencias presentadas en el índice de masculinidad puede encontrarse en la muestra de cada una de las poblaciones estudiadas, ya que la utilizada por la paleodemografía consiste tan sólo en un pequeño número de individuos de los que se enterraron a lo largo de casi un siglo en el panteón, lo que imprime un sesgo a las cantidades de individuos por sexo y grupos de edad; además obedece a la propia probabilidad de muerte diferencial por sexo y grupo de edad, pues eran más las probabilidades de morir en las primeras edades para los hombres, y para las mujeres en las edades reproductivas.

En cuanto al análisis exploratorio del sistema logito, en ninguno de los dos casos se ajusta completamente a la recta, por lo que este medio exploratorio muestra a simple vista las deficiencias de los datos que integran las muestras poblacionales estudiadas por los demógrafos y antropólogos interesados en el tema.

Los resultados obtenidos en el análisis demográfico no son comparables, ya que difieren en cada uno de los indicadores de mortalidad y fecundidad; así, presentan valores más altos en los casos de la tasa de mortalidad infantil, la tasa bruta de mortalidad, la tasa bruta de natalidad y las tasas específicas de fecundidad; al igual que los valores de $d_{x}$ al graficarlos en la curva de mortalidad en la demografía histórica, mientras que la paleodemografía solamente los presenta en la edad media a la muerte y la esperanza de vida al nacimiento. 
Horowitz et al. (1988) expusieron que la distribución de las muertes en cada grupo de edad, más que un reflejo de la mortalidad lo es de la fecundidad, por ello al haber mayor número de defunciones en los grupos de edades jóvenes, el cálculo directo de la fecundidad se muestra alto y los valores de la edad media a la muerte y la esperanza de vida son menores; tal es el caso presentado en la Parroquia de Santa María la Redonda. Por lo mismo al realizarse el cálculo de la fecundidad con valores menores, la esperanza de vida y la edad media a la muerte alcanzan valores más altos, como fue el caso de la paleodemografía.

El presente estudio comparativo nos muestra dos escenarios posibles donde la fecundidad se observa alta a partir del análisis de la demografía histórica y baja a partir de la paleodemografía, lo cual tiene implicaciones en el cálculo indirecto de la mortalidad de una misma población en un mismo momento sociohistórico.

Ello nos lleva a formular las siguientes preguntas: ¿Son compatibles los resultados como para unificarlos en una sola inferencia demográfica respecto a las poblaciones humanas del pasado?, ¿o simplemente pueden mostrarnos rangos de valores posibles de las tasas e índices empleados por la demografía? Si dejamos de lado las diferencias generadas primordialmente por la calidad y cantidad de las muestras por edad y sexo observadas en ambos métodos, y unificamos el estudio a partir de lo mejor de cada uno de ellos, cabría cuestionar si realmente se puede obtener de esta unificación el contexto demográfico completo e ideal para comprender las sociedades del pasado. Sin embargo la interrogante relativa a la unificación de estudios cuyos valores sean rangos entre los que podrían oscilar los fenómenos demográficos queda a mi juicio sin respuesta convincente; empero, tal método podría ser empleado de acuerdo con el criterio del investigador y según el contexto histórico y social de la población en estudio.

Esta crítica reflexiva nos lleva, tras presentar los dos escenarios, a concluir que la idea de complementar el estudio de las poblaciones del pasado valiéndonos de dos disciplinas y de sus fuentes de información (que son diametralmente diferentes por los supuestos metodológicos con que trabajan y por los tipos de fuentes de los que parten) no es válida, porque de inicio no es posible que en un mismo momento sociohistórico haya dos comportamientos de fecundidad a partir de poblaciones contemporáneas, y por tanto esa complementariedad, más que ayudarnos a tener una idea precisa del comportamiento de nuestras poblaciones, hayan tenido o no fuentes es- 
critas, puede llevarnos a confundir lo que realmente estaba pasando en cuanto a la mortalidad, la fecundidad y la migración.

\section{Bibliografía}

Aguirre, Alejandro (1998), "El efecto del descenso de la fecundidad en la estructura por edad de la población", en Héctor Hiram Hernández Bringas y Catherine Menkes (coords.), La población de México al final del siglo XX (V Reunión de Investigación Sociodemográfica en México), vol. 1, México, Sociedad Mexicana de Demografía/ Centro Regional de Investigaciones Multidisciplinarias, Universidad Nacional Autónoma de México, pp. 25-44.

Arretx Gutiérrez, Carmen, Rolando Mellafe y Jorge L. Somoza (1983), Demografía histórica en América Latina: fuentes y métodos, San José, Costa Rica, Centro Latinoamericano de Demografía (Celade).

Bass, William M. (1987), Human Osteology: a Laboratory and Field Manual, Columbia, Mo., Missouri Archaeological Society (Special Publication, 2).

Boquet-Appel, J. P. y C. Masset (1982), "Farewell to Paleodemography", Journal of Human Evolution, vol. 11, pp. 321-333.

- (1985), "Paleodemography: Resurrection or Ghost?", Journal of Human Evolution, vol. 14, pp. 107-111.

Brass, William (1977), "Notes on Empirical Mortality Models", Population Bulletin of the United Nations, núm. 9.

Buikstra, Jane E. y Lyle W. Konigsberg (1985), "Paleodemography: Critiques and Controversies", American Anthropologist, vol. 87, núm. 2, pp. 316-333.

Bustamante, Miguel E. (1982), "Cronología epidemiológica mexicana en el siglo xIx", en Enrique Florescano y Elsa Malvido (comps.), Ensayo sobre la historia de las epidemias en México, t. 2, México, Imss (Colección Salud y Seguridad Social), pp. 417-424.

Ceja Moreno, Mario y Patricia O. Hernández Espinoza (1998), "Algunos datos demográficos de la población novohispana de la Ciudad de México", en Lourdes Márquez Morfín y José Gómez de León (comps.), Perfiles demográficos de poblaciones antiguas de México, México, INAH (Colección Obra Diversa), pp. 126-154.

Celade, Comisión de Historia Económica, Clacso (1975), Fuentes para la demografía histórica de América Latina, México, Departamento de Investigaciones Históricas, INAH (Cuadernos, 2).

Chena R., Rodolfo (1992), "La población de una parroquia novohispana del siglo xviII: Santa María de la Presentación de Chilapa”, Estudios Demográficos y Urbanos, vol. 7, núm. 1 (19), pp. 169-192.

Civera Cerecedo, Magali y Lourdes Márquez Morfín (1998), "Paleodemografía: sus alcances y limitaciones”, en Lourdes Márquez Morfín y José 
Gómez de León (comps.), Perfiles demográficos de poblaciones antiguas de México, México, InAH (Colección Obra Diversa), pp. 15-25.

Coale, A. J. y J. P. Demeny (1983), Regional Model Life Tables and Stables Populations, Nueva York, Academic Press (segunda edición).

Dupâquier, Jacques (1984), Pour la démographie historique, París, Presses Universitaires de France.

Ens, Gerhard J. (1996), Homeland to Hinterland. The Changing Worlds of the Red River Metis in the Nineteenth Century, Toronto, University of Toronto Press.

Florescano, Enrique y Margarita Menegus (2000), "La época de las reformas borbónicas y el crecimiento económico (1750-1808)", en Historia general de México (versión 2000), México, Centro de Estudios Históricos, El Colegio de México, pp. 363-430.

Gómez de León, José (1998), “Análisis paleodemográfico de poblaciones antiguas de México: algunas estimaciones y comentarios metodológicos”, en Lourdes Márquez Morfín y José Gómez de León (comps.), Perfiles demográficos de poblaciones antiguas de México, México, INAH (Colección Obra Diversa), pp. 155-170.

Henry, Louis (1968), "Historical Demography”, Daedalus, vol. 97, núm. 2, pp. 415-433.

— y M. Fleury (1956), Des registres paroissi aux à l'histoire de la population: manuel de dépouillement et d'exploitation de l'état civil ancien, París, INED.

Hernández Espinoza, Patricia O. (1999), "La paleodemografía en México", Revista Argentina de Antropología Biológica, núm. 1.

Hollingsworth, T. H. (1968), "The Importance of the Quality of the Data in Historical Demography”, Daedalus, vol. 97, núm. 2, pp. 415-433.

Horowitz, Sheryl, George Armelagos y Ken Wachter (1988), "On Generating Birth Rate from Skeletal Populations", American Journal of Physical Anthropology, vol. 76, pp. 189-196.

Johansson, Ryan S. y Sheryl Horowitz (1986), "Estimating Mortality in Skeletal Populations: Influence of the Growth Rate on the Interpretation of Levels and Trends During the Transition to Agriculture", American Journal of Physical Anthropology, vol. 71, pp. 233-250.

Kicza, John E. (1993), "Historia demográfica mexicana del siglo xIx: evidencias y aproximaciones”, en Elsa Malvido y Miguel Ángel Cuenya (comps.), Demografía histórica de México: siglos XVI-XIX, México, Instituto Mora/Universidad Autónoma Metropolitana, pp. 217-262.

Konigsberg, Lyle W. y Susan R. Frankenberg (1992), "Estimation Structure in Anthropological Demography", American Journal of Physical Anthropology, vol. 89, pp. 235-256.

Lagunas Rodríguez, Zaíd y Patricia O. Hernández Espinoza (2000), Manual de osteología, México, Conaculta/ENAH, INAH.

Le Goff, Jaques (1997), Pensar la historia: modernidad, presente, progreso, Barcelona, Paidós. 
Lovejoy, C. Owen, Richard S. Meindl, T. R. Pryzbeck y Robert P. Mensforth (1985), "Chronological Metamorphosis of the Auricular Surface of the Ilium: a New Method for the Determination of Adulth Skeletal Age at Death”, American Journal of Physical Anthropology, vol. 68, pp. 15-28.

Maldonado L., Celia (1978), "Edad promedio a la muerte, Ciudad de México, 1840-1849”, México, Departamento de Investigaciones Históricas, INAH (Cuaderno de Trabajo, 22), pp. 157-158.

(1976), Estadísticas vitales de la Ciudad de México (siglo XIX), México, Seminario de Historia Urbana, Departamento de Investigaciones Históricas, INAH (Colección Científica, 31).

Márquez Morfín, Lourdes (1998), "Los parroquianos del Sagrario Metropolitano: panorama sociodemográfico”, en Lourdes Márquez Morfín y José Gómez de León (comps.), Perfiles demográficos de poblaciones antiguas de México, México, inaH (Colección Obra Diversa), pp. 95-125.

— (1992), "El cólera en la Ciudad de México en el siglo xıx", Estudios Demográficos y Urbanos, vol. 7, núm. 1 (19), pp. 77-94.

—_ y Patricia O. Hernández Espinoza (2001), Principios básicos, teóricos y metodológicos de la paleodemografía, México, INAH, ENAH.

McCaa, Robert (2000), "Paleodemography of the Americas: From Ancient Times to Colonialism and Beyond", en Richard H. Steckel y Jerome Rose (eds.), The Backbone History: Long-Term Trends in Health and Nutrition in the Americas (en prensa, versión del 1 de diciembre de 2000).

Meindl, Richard S. y Katherine F. Russell (1998), "Recent Advances in Method and Theory in Paleodemography", Annual Review of Anthropology, vol. 27, pp. 375-399.

$\longrightarrow$ y C. Owen Lovejoy (1985), "Ectocranial Suture Closure: a Revised Method for the Determination of the Skeletal Age at Death Based on the Lateral-Anterior Sutures", American Journal of Physical Anthropology, vol. 68 , pp. 57-66.

Moore, J. A., A. C. Swedlund y G. J. Armelagos (1975), "The Use of Life Tables in Paleodemography", Population Studies in Archaeology: a Symposium, American Antiquity, vol. 40, núm. 2.

Müller, Hans-Georg, Brad Love y Robert D. Hoppa (2002), "Semiparametric Method for Estimating Paleodemographic Profiles from Age Indicator", American Journal of Physical Anthropology, vol. 117, pp. 1-14.

Oliver, Lilia V. (1992), “La mortalidad, 1800-1850”, en José María Muriá y Jaime Olveda (comps.), Demografía y urbanismo: lecturas históricas de Guadalajara III, Guadalajara, Programa de Estudios Jalisciences/INAH/Gobierno del Estado de Jalisco/Universidad de Guadalajara, pp. 101-140.

Ortega Muñoz, Allan (2002), Demografía histórica y paleodemografía: un análisis comparativo del comportamiento demográfico en la Ciudad de México, siglo XIX, tesis de maestría en Demografía, México, El Colegio de México.

- (1998), La estimación de edad en restos óseos subadultos mesoamericanos. Colección osteológica de San Gregorio Atlapulco, Xochimilco, tesis de licencia- 
tura en Antropología Física, México, Escuela Nacional de Antropología e Historia.

Paine, Richard R. (2000), "If a Population Crashes in Prehistory, and There Is No Paleodemographer There to Hear It, Does It Make a Sound?", American Journal of Physical Anthropology, vol. 112, pp. 181-190.

Pescador Cantón, Juan Javier (1992a), "La nupcialidad urbana preindustrial y los límites del mestizaje: características y evolución de los patrones de nupcialidad en la Ciudad de México, 1700-1850”, Estudios Demográficos y Urbanos, vol. 7, núm. 1 (19), pp. 137-168.

- (1992b), De bautizados a fieles difuntos: familia y mentalidades en una parroquia urbana: Santa Catarina de México, 1568-1820, México, El Colegio de México.

Piontek, J. y A. Weber (1990), "Controversy on Paleodemography", International Journal of Anthropology, vol. 5, núm. 1, pp. 71-83.

Revelle, Roger (1968), "Introduction to the Issue 'Historical Population Studies'”, Daedalus, vol. 97, núm. 2, pp. 353-362.

Rodríguez, Jaime (1986), "La crisis de México en el siglo xIx", Estudios de Historia Moderna y Contemporánea de México, México, Instituto de Investigaciones Históricas, Universidad Nacional Autónoma de México, pp. 85107.

Tuirán Gutiérrez, Rodolfo (1992), "Algunos hallazgos recientes de la demografía histórica mexicana”, Estudios Demográficos y Urbanos, vol. 7, núm. 1 (19), pp. 273-312.

Velasco M. L., María del Pilar (1992), "La epidemia de cólera de 1833 y la mortalidad en la Ciudad de México”, Estudios Demográficos y Urbanos, vol. 7, núm. 1 (19), pp. 95-137.

Walker, Phillip L., John R. Johnson y Patricia M. Lambert (1988), “Age and Sex Biases in the Preservation of Human Skeletal Remains", American Journal of Physical Anthropology, vol. 76, pp. 183-188.

Wood, James W., George R. Milner, Henry C. Harpending y Kenneth M. Weiss (1992), "The Osteological Paradox. Problems of Inferring Prehistoric Health from Skeletal Samples”, Current Anthropology, vol. 33, núm. 4, pp. 343-370.

Wunsch, Guillaume (1992), Técnicas para el análisis de datos demográficos deficientes, México, El Colegio de México. 